dense left hemiparesis developed. She died 2 years 7 months after the onset of seizures. (Harvey AS, Andermann F et al. Chronic encephalitis (Rasmussen's syndrome) and ipsilateral uveitis. Ann Neurol Dec 1992; 32: 826-829). (Correspondence: Dr Harvey, Dept of Neurology, Royal Children's Hospital, Flemington Rd, Parkville, Victoria 3052, Australia).

COMMENT. A viral cause seemed likely but was not confirmed by serology or tissue culture. Slit-lamp examination of the eye should be included in the evaluation of children with Rasmussen's syndrome.

\title{
ENCEPHALOPATHIES
}

\section{ACUTE ENCEPHALOPATHY OF OBSCURE ORIGIN}

Six previously healthy children who developed an acute encephalopathy several days after a prodromal illness are reported from the Hopital Bicetre, and Hopital Necker-Enfants Malades, Paris, France. Prodromal illnesses consisted of upper respiratory infection with fever, and headache and vomiting. Coma was the initial symptom in 4 patients. Abnormal movements included gesticulation, chewing, swallowing, orofacial dyskinesia, limb dystonia, and choreoathetosis. Rigidity was constant in 5 patients and intermittent in one. Seizures occurred in 2 patients. Recovery extended for several weeks and was characterized by a rapid return of motor function and persistent behavioral and cognitive disturbances. Four patients recovered fully, and two had mild sequelae. (Sebire G et al. Coma associated with intense bursts of abnormal movements and long-lasting cognitive disturbances: An acute encephalopathy of obscure origin. L Pediatr Dec 1992; 121: 845-51). (Reprints: G Sebire MD, Service de Neurologie, Departement de Pediatre, Hopital Bicetre, 78 rue du general Leclerc, 94275, Le Kremlin Bicetre Cedex, France).

COMMENT. These cases with a favorable outcome were thought to represent a different syndrome from that described by Lyon, Dodge, and Adams, whose 16 patients died from an acute encephalopathy of obscure origin. Attempts at viral isolation and antibody detection were negative.

\section{DEGENERATIVE DISEASES}

\section{HEPATOCEREBRAL DEGENERATION OR VALPROATE TOXICITY}

Six children with refractory seizures and focal neuronal damage who died of liver failure are reported from the Washington University School of Medicine, St Louis, MO. Four were treated with valproic acid (VPA) and developed liver failure within $30-68$ days. Two of these children each had one sibling who was not exposed to VPA and developed the same clinical picture, but liver failure was delayed. Siblings receiving VPA survived only 3 and 5 